\title{
Analysis of the chromosomal localization of the repetitive sequences (RPSs) in Candida albicans
}

\author{
Ariya Chindamporn, Yoshiyuki Nakagawa, Michio Homma, $†$ \\ Hiroji Chibana, Matsuko Doił and Kenji Tanaka
}

Author for correspondence: Y. Nakagawa. Tel: +81527412111 ext. 2115. Fax: +81527319479.
e-mail : e43187a@nucc.cc.nagoya-u.ac.jp

Laboratory of Medical Mycology, Research Institute for Disease Mechanism and Control, Nagoya University School of Medicine, Showa-ku, Nagoya 466, Japan

\begin{abstract}
The location and organization of repetitive sequences, members of the RPS family, which are sequences specific to Candida albicans, were determined on each chromosome of C. albicans strain FC18. Using pulsed-field gel electrophoresis, we separated seven fractions from eight chromosomes. Each chromosome was cleaved by BamHI and Xhol to excise the RPSs, which were then detected by hybridization with an RPS probe. All chromosomes except chromosome 4 carried RPSs, and these RPSs were located within a limited region on each chromosome. From the digestion of each chromosome with Sfil and probing with the RPSs, we found that these recognition sites within the RPS region were conserved among all RPS-containing chromosomes. For further characterization of the RPSs, the locations and the boundary regions of the RPSs were examined on chromosome 6 of strain FC18 as a model chromosome. Using the restriction enzymes Sfil, Smal, Xhol, BamHI, Mlul and Nrul, we constructed a semi-macro physical map of the RPSs and their boundary regions on this chromosome. We also determined which part of the RPS was adjacent to each boundary by using sub-fragments of RPS as probes. The physical configuration around the RPSs and their boundary regions are presented. The results obtained should be useful for future analysis of the function of these regions.
\end{abstract}

Keywords: repetitive sequence, RPS, physical map, Candida albicans

\section{INTRODUCTION}

The recent development of techniques, such as pulsedfield gel electrophoresis (PFGE; Schwartz \& Cantor, 1984), for manipulating high-molecular-mass DNA has revealed that hyper-variability in chromosomal features is one of the most distinctive characteristics of Candida albicans (Lott et al., 1987; Magee \& Magee, 1987; Iwaguchi et al., 1990; Doi et al., 1992). In some cases, changes in karyotype are known to be associated with a variation in some aspect of the phenotype, such as colony morphology or cell shape, which can be correlated with the expression of pathogenicity in this yeast (Suzuki et al., 1989;

\footnotetext{
† Present address: Department of Molecular Biology, Faculty of Science, Nagoya University, Chikusa-ku, Nagoya 464-01, Japan.

$\ddagger$ Present address: Gifu University College of Medical Technology, Kitanomachi, Gifu 500, Japan.
}

Abbreviation: PFGE, pulsed-field gel electrophoresis.
Rustchenko-Bulgac et al., 1990; Barton \& Scherer, 1994). Repeated sequences in $C$. albicans that might be associated with chromosomal variations or translocations have been isolated in several laboratories (Scherer \& Stevens, 1988; Lasker et al., 1989, 1991; Sadhu et al., 1991; Iwaguchi et al., 1992a; Thrash-Bingham \& Gorman, 1993). Some of these sequences are known to be species-specific and can serve as useful probes for the epidemiological analysis of clinical isolates (Scherer \& Stevens, 1988; Anderson et al., 1993). However, little is known about the precise configuration of these repetitive sequences in the genome of this yeast or about their possible functional significance.

The most variable, in terms of size, of the eight chromosomes of $C$. albicans was reported previously to be chromosome 2 , followed by chromosome 6 . A change in the number of repeating units of ribosomal DNA is responsible for the variability in length of chromosome 2 (Iwaguchi et al., 1992b). Another repetitive sequence, RPS1, was cloned from genomic DNA of C. albicans strain 
NUM812 and was characterized. It hybridized to chromosome 6 of strain FC18 and also to other chromosomes (Iwaguchi et al., 1992a). Sequences homologous to the RPS1 sequence from different chromosomes of strain FC18 have been designated members of the RPS family, including RPS1 (Chibana et al., 1994). Sequence analysis revealed that members of the RPS family are composed of a few sequence repeats (172 bp) known as alt, which contain shorter, common stretches known as COM29 (29 bp; Iwaguchi et al., 1992a; Chibana et al., 1994). This structural hierarchy of the repeating sequences is very similar to the structure of sequences believed to be involved in centromeric functions in humans (Willard \& Waye, 1987). Thus, characterization of the chromosomal locations of RPSs is a prerequisite for studies of the function of RPS.

In this paper, we demonstrate that RPSs are located on every chromosome, except chromosome 4 , of $C$. albicans and are clustered in a limited region on each chromosome. We also provide a detailed analysis of chromosome 6 , which is the second most variable in size of this yeast's chromosomes. Analysis of the SfiI digestion profile revealed that RPSs are clustered in the middle region of chromosome 6 . To clarify the precise position of the RPSs, a semi-macro physical map of RPSs and their boundary regions on chromosome 6 was constructed.

\section{METHODS}

Strains and plasmids. C. albicans strains FC18, NUM46, NUM55 and NUM1039 were used (Iwaguchi et al., 1990, 1992a). The homologue of chromosome 6 from each of the three NUM strains was isolated (see below). The plasmids used as sources of probe DNAs for Southern hybridization were as follows: pSI3-12 containing an RPS1 from $C$. albicans strain NUM812 (Iwaguchi et al., 1992a); pRPS101, pRPS116 and pRPS620 containing RPS101, RPS116 from chromosome $1+2$ and RPS620 from chromosome 6 of strain FC18, respectively (Chibana et al., 1994); pMM100 containing a telomeric sequence of Ca7 (Sadhu et al., 1991; McEachern \& Hicks, 1993); and pTK2-9-1 containing the chromosome 6 marker of $C$. albicans (Magee et al., 1988; Iwaguchi et al., 1990, 1992b).

Preparation of chromosomal DNA for PFGE. The sample plug containing yeast chromosomal DNA for PFGE was prepared by the method described previously (Iwaguchi et al., 1990). PFGE was carried out by the CHEF method using the Pulsaphor system with a hexagonal electrode array (PharmaciaLKB) as described previously (Iwaguchi et al., 1992a). Chromosomes of Saccharomyces cerevisiae (X2180-1A), $\lambda$ phage ladder concatemeric DNA (Bio-Rad) and $\lambda$ phage DNA digested by EcoT14I (Takara Shuzo) were used as molecular-size markers.

Southern hybridization. Southern hybridization was performed by using a probe prepared from an EcoRI fragment of pSI3-12 or a PstI fragment of pRPS101 for detection of RPSs and an EcoRI fragment of pTK2-9-1 for assignment of chromosome 6 (Iwaguchi et al., 1990, 1992a; Chibana et al., 1994). The probes were prepared by random primer labelling with $\left.{ }^{32} \mathrm{P}\right] \mathrm{dCTP}$ and hybridization signals were detected by autoradiography on X-ray film or by an image analyser (BAS 2000; Fuji Photo Film).

Restriction digestion of total chromosomal DNA in sample plugs for PFGE. This was performed as described previously (Chindamporn et al., 1993).
BamHI and Xhol digestion of individual chromosomes. Each chromosome of $C$. albicans strain FC18 was separated from total chromosomal DNA (sample plug) on $0.7 \%$ (w/v) gels by PFGE under conditions of $100 \mathrm{~s}$ at $170 \mathrm{~V}$ for $15 \mathrm{~h}, 300 \mathrm{~s}$ at $130 \mathrm{~V}$ for $24 \mathrm{~h}$ then $1000 \mathrm{~s}$ at $80 \mathrm{~V}$ for $48 \mathrm{~h}$. After staining the gels with ethidium bromide and destaining with distilled water, individual bands corresponding to each chromosome were cut out from the gel under UV light $(365 \mathrm{~nm})$. To perform endonuclease digestion, the ethidium bromide was removed from the gel slices (about $2 \mathrm{~mm}^{3}$ in size) by three 10 min washes with sec-butanol saturated with $1 \mathrm{M} \mathrm{NaCl}, 10 \mathrm{mM}$ Tris/ $\mathrm{HCl}$ (pH 8.0) and $1 \mathrm{mM}$ EDTA. The samples were then equilibrated in $200 \mu \mathrm{l}$ of the appropriate restriction endonuclease buffer for $30 \mathrm{~min}$, transferred into $200 \mu \mathrm{l}$ fresh buffer with either $10 \mathrm{U}$ Bam HI or $10 \mathrm{U} X b o \mathrm{I}$ (Takara Shuzo) and incubated overnight at $37^{\circ} \mathrm{C}$. The digested products were separated by subsequent electrophoresis (see legend of Fig. 1) prior to hybridization analysis using RPS1 probes.

Restriction digestion of each chromosome 6 that had been separated by PFGE. Chromosome 6 of strain FC18 was prepared as described above. The gel slices were equilibrated in $200 \mu \mathrm{l}$ of the appropriate restriction endonuclease buffer for $30 \mathrm{~min}$. They were then transferred into $200 \mu \mathrm{l}$ fresh buffer, the appropriate restriction enzyme added $20 \mathrm{U}$ S fiI, $10 \mathrm{U}$ NruI (New England Biolab), $10 \mathrm{U}$ BamHI, $10 \mathrm{U}$ MluI, $10 \mathrm{U}$ SmaI or $10 \mathrm{U}$ XhoI (Takara Shuzo)] and incubated overnight under the appropriate optimum conditions as indicated by the manufacturers.

\section{RESULTS}

\section{Localization of RPSs on individual chromosomes}

Since no recognition sites for $\mathrm{XboI}, \mathrm{BamHI}$ and SalI were found within RPSs, these enzymes were suitable for separation of RPS-containing regions from each chromosome. The pattern of digestion by $X b o \mathrm{I}$ or BamHI was revealed by PFGE and subsequent Southern hybridization with probe pSI3-12 (Fig. 1a, b). The digestion profiles obtained with both these enzymes from whole chromosomes (sample plugs prepared for PFGE) revealed 10-15 bands after hybridization with an RPS probe (Fig. 1a, b, lane Total). This hybridization profile contained bands which corresponded to the hybridization bands derived from the different individual chromosomes (lanes $1+2$ to 8). For example, in the case of digestion with BamHI (Fig. 1a), the bands of 50,102, and 120 plus $102 \mathrm{kbp}$ were derived from chromosomes 2, 3 and 6, respectively. Between one and four bands from individual chromosomes hybridized to the RPS probe. If we assume that the FC18 strain carries two homologues for every chromosome, one band would be obtained from each chromosome if the cleavage sites for a restriction enzyme that flank the RPS-containing region are conserved between the two homologues. However, when two bands are detected there are two possible locations of the RPSs: the RPSs might be located at two different loci on a chromosome, or they might be located at a single locus on a chromosome but the cleavage sites for the restriction enzymes used are not in the same position. To determine whether or not there is only one RPS(s) locus on a chromosome, we used strains in which the two homologues of chromosome 6 are separable by PFGE. After separation and isolation of chromosome 6 following gel electrophoresis, the samples were digested with $\mathrm{XboI}$ and 

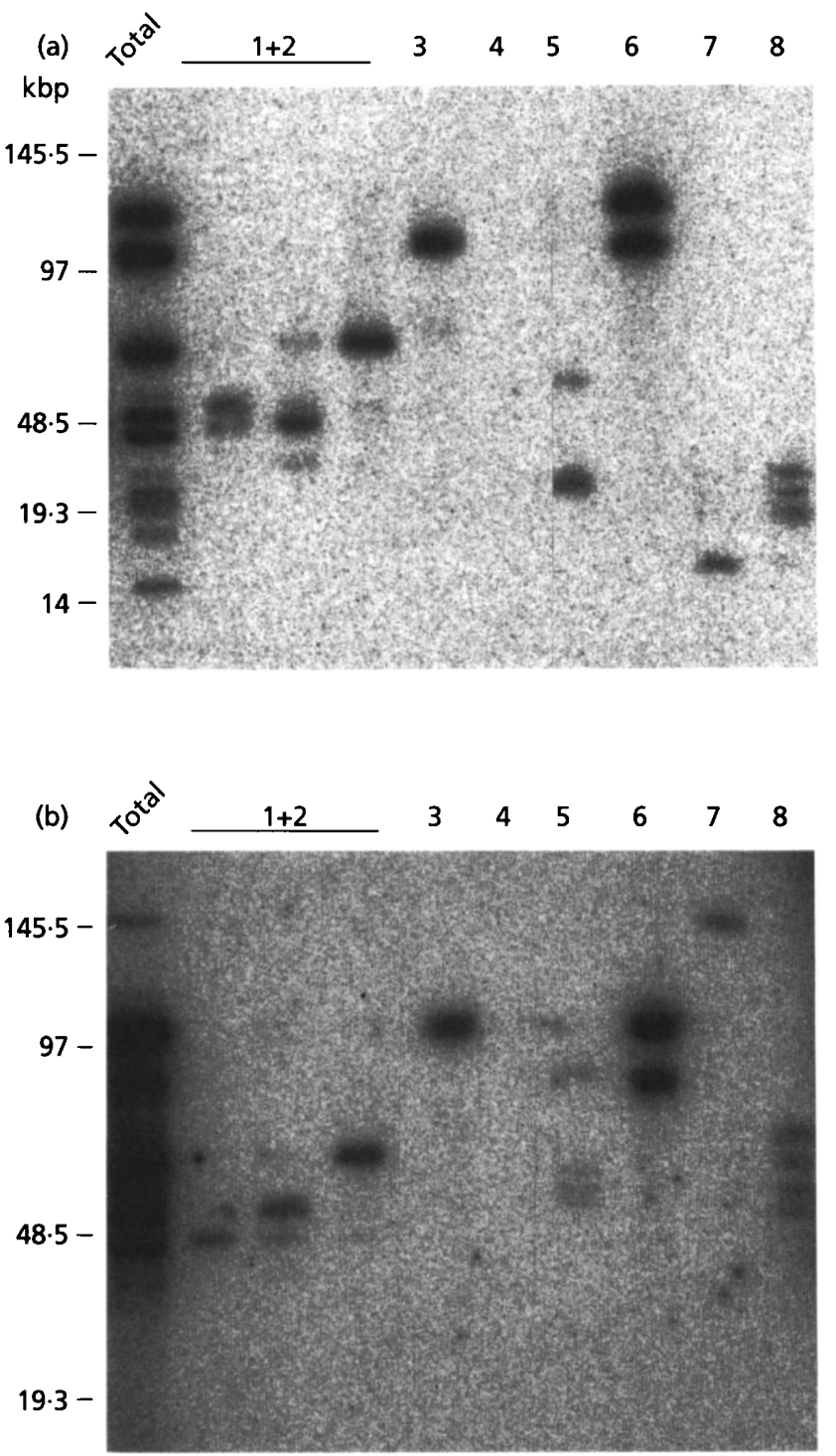

Fig. 1. Distribution of RPSs on the chromosomes of $C$. albicans FC18. Each of the chromosomes separated by PFGE and the total genomic DNA (see Methods) were digested with BamHI (a) or Xhol (b), and subjected to PFGE followed by Southern hybridization with a ${ }^{32}$ P-labelled RPS1 as an RPS probe. The digested samples were separated by PFGE under the following conditions: linear ramping of $2.5-12.5 \mathrm{~s}$ at $200 \mathrm{~V}$ for $20 \mathrm{~h}$ on $1.2 \%(w / v)$ agarose. Molecular sizes were estimated from the mobilities of $\lambda$ phage concatemeric DNA (Bio-Rad) that had been labelled with ${ }^{32} \mathrm{P}$. The leftmost lane (Total) indicates the digested total genomic DNA, and the other lanes indicate the digested individual chromosomes. The numbers at the top of the lanes represent the chromosome numbers. Chromosomes 1 and 2 were not clearly separated under the conditions used here. Data shown in the figure were from one set of experiments. Similar results were obtained in separate experiments.

the resulting fragments separated by PFGE and analysed by Southern hybridization. As shown in Fig. 2, only one hybridization band was detected from each separated homologue, although the mobility of the band varied from chromosome to chromosome with different strains.

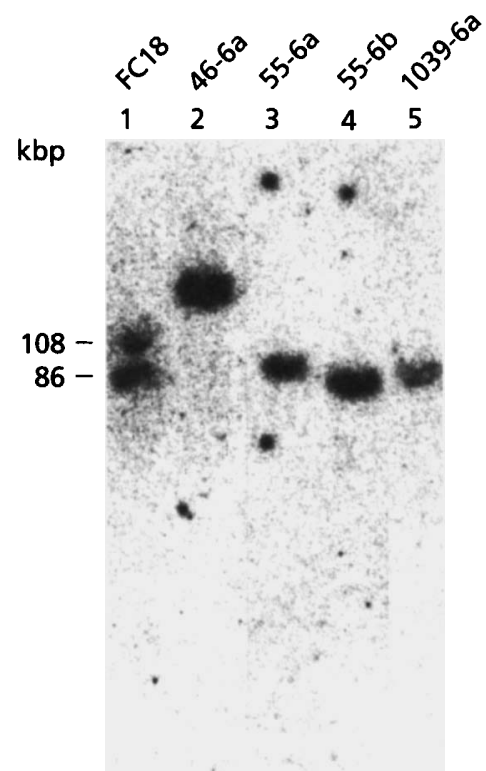

Fig. 2. The RPS hybridization profile of Xhol fragments of chromosome 6 from various C. albicans strains. Chromosomal DNA bands corresponding to chromosome 6 of each strain used were prepared and their identity confirmed by probe PTK 2-9-1 (Iwaguchi et al., 1990; A. Chindamporn, unpublished results). The DNA in gel slices containing the individual bands was digested with Xhol (see Methods). After treatment with this enzyme, samples were subjected to PFGE with ramping conditions of $5-20 \mathrm{~s}$ at $200 \mathrm{~V}$ for $20 \mathrm{~h}(1.25 \%, \mathrm{w} / \mathrm{v}$, agarose $)$ and analysed by Southern hybridization using the ${ }^{32}$ P-labelled RPS1 as a probe. Lanes: 1 (FC18), the homologues of FC18; 2 (46-6a), the large homologue of NUM46; $3(55-6 a)$, the large homologue of NUM55; 4 (55-6b), the small homologue of NUM55; 5 (1039$6 a)$, the large homologue of NUM1039. The numbers on the left side are molecular sizes calculated from mobilities of chromosomes of $S$. cerevisiae and $\lambda$ phage concatemers. Data shown in the figure were from one set of experiments. Similar results were obtained in separate experiments.

Similar results were obtained when $M l u \mathrm{I}$ or $\mathrm{NruI}$ was used as the restriction enzyme (data not shown). In the case of chromosome 8 , which yielded four bands after digestion with $X b o \mathrm{I}$ or BamHI (Fig. 1a, b; lane 8), a site susceptible to cleavage by these enzymes might exist within the RPS region, or the RPS-containing regions on the two homologues might be of two different lengths. However, when SalI was used instead of these two enzymes, only two RPS-positive bands were detected (data not shown). This result indicates that the RPS region on chromosome 8 might contain $B a m \mathrm{HI}$ and $\mathrm{XboI}$ recognition sites and, as a result, four bands were detected. Thus, the RPS region on this chromosome was also shown to be located in a limited region. Another interesting finding was the failure to detect a hybridization signal with the RPS probe on chromosome 4 (Fig. 1a, b, lane 4), even after both longer exposure of hybridization filters to X-ray film and low-stringency conditions of hybridization were performed (data not shown). Nevertheless, the possible presence of very short stretches homologous to the RPS cannot be excluded. 


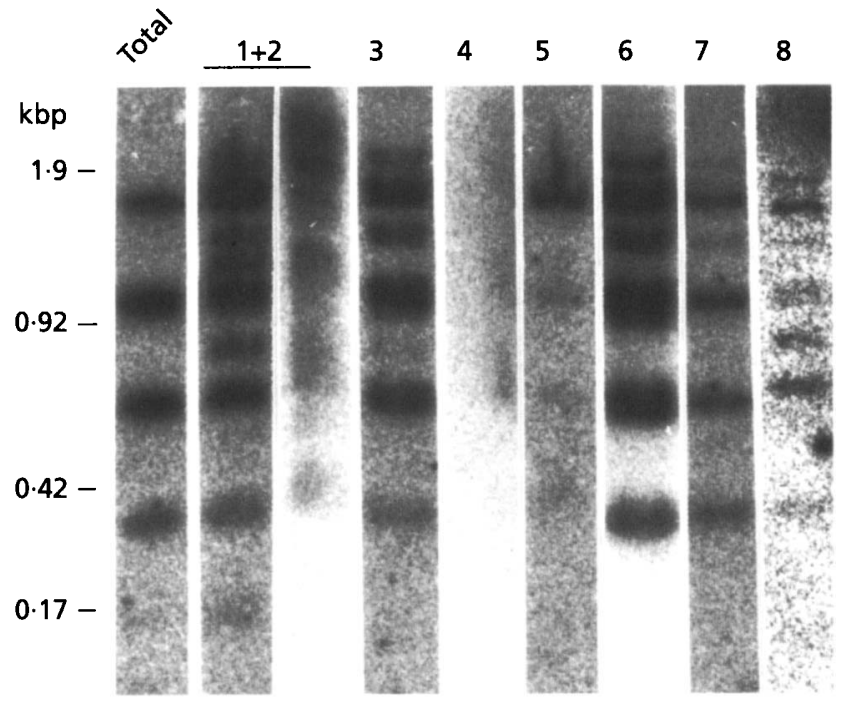

Fig. 3. Detection of small-sized fragments after Sfil treatment of each chromosome. After separation by PFGE, each chromosome in the $0.7 \%(\mathrm{w} / \mathrm{v})$ agarose gel was isolated and transferred to $0.4 \%(\mathrm{w} / \mathrm{v})$ agarose with subsequent treatment with Sfil (see Methods). The digested samples were fractionated by electrophoresis and subjected to Southern hybridization using a mixture of pRPS101, pRPS116 and pRPS620 (Chibana et al., 1994) as the source of the probe for RPSs. EcoT14l-treated $\lambda$ phage and Hinfl-treated bacteriophage $\phi$ X174 DNA were used as molecular size markers, as shown on the left. The leftmost lane (Total) represents Sfil-digested total genomic DNA, and the respective lanes indicate digested individual chromosomes. The numbers at the top of these lanes represent the chromosome numbers (Iwaguchi et al., 1990). Chromosomes 1 and 2 were not clearly separated under the conditions used here. Data shown in the figure were from one set of experiments. Similar results were obtained in separate experiments.

From our characterization of the RPS(s), each RPS unit was shown to have several $S$ fi sites in all clones derived from chromosomes $1+2$ and 6 (Chibana et al., 1994). In order to obtain information about whether or not the RPSs on the other chromosomes also contain such recognition sites, each chromosome was digested by this enzyme. As shown in Fig. 3, all chromosomes apart from chromosome 4 gave signals that corresponded to about $0 \cdot 34,0.68$ and $1.1 \mathrm{kbp}$, namely, the sizes expected from the digestion by Sfil of the RPS, although the intensity of bands was not uniform. In addition the fragment of about $1.8 \mathrm{kbp}$ in size, which was not identified from our RPS clones, was commonly detected from total genomic DNA and fractionated chromosomes except chromosome 4. The other bands observed in fractionated chromosomes were likely to be as a result of partial digestion with SfII. Moreover, a $170 \mathrm{bp}$ fragment, corresponding to the size of some RPSs (Chibana et al., 1994), was detected in the analysis of chromosomes $1+2$ and 8 following a longer exposure time after hybridization. This fragment was also detected in the analysis of total genomic DNA, although the signals were very weak. Thus, our results strongly suggest that all of the chromosomes except chromosome 4 include RPSs that are present within limited regions on

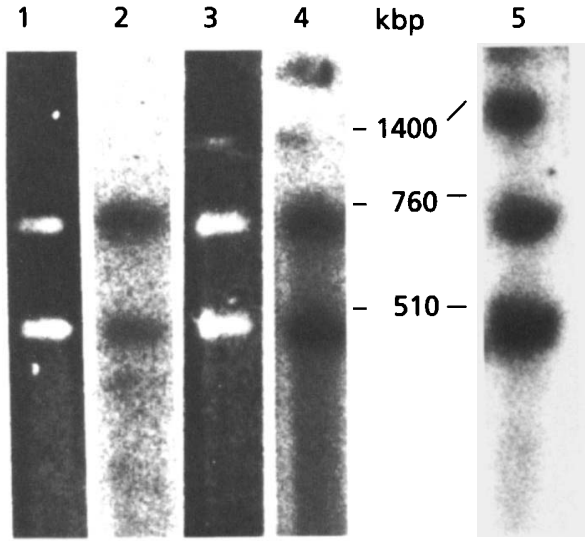

Fig. 4. Southern hybridization profile of the Sfil fragments from chromosome 6 by using pSI3-12 and pMM100 as probes. The isolated chromosome 6 of FC18 following recovery from a PFGE gel was treated with Sfil (see Methods) and the digested samples fractionated by PFGE under ramping conditions of $20-100 \mathrm{~s}$ at $180 \mathrm{~V}$ for $15 \mathrm{~h}$ on a $1 \%(\mathrm{w} / \mathrm{V})$ agarose gel. Results of complete digestion with Sfil are shown in lanes 1 and 2; results of partial digestion are shown in lanes 3 and 4 . Lanes 1 and 3, ethidium bromide staining; lanes 2 and 4, Southern hybridization with the ${ }^{32}$ P-labelled RPS1 as a probe. Lane 5 shows the results of Southern hybridization with a ${ }^{32} \mathrm{P}$-labelled telomeric sequence from pMM100 as a probe (Sadhu et al., 1991; McEachern \& Hicks, 1993). Data shown in the figure were from one set of experiments. Similar results were obtained in separate experiments.

the respective chromosomes. However, further investigation of the possible presence of RPS on chromosome 4 is necessary.

\section{Analysis of the boundary regions of RPSs}

To elucidate the biological functions of RPS, we constructed a plasmid that contained RPS620 cloned in the C. albicans vector pMK22 containing the Candida autonomously replicating sequence (CARS) and the $U R A 3$ gene (Kurtz et al., 1987). Using this plasmid, we transformed $\mathrm{Ura}^{-}$C. albicans strains such as 1006 and SGY243 to yield $\mathrm{Ura}^{+}$transformants. However, no transformants which contained a stable episome were obtained. Therefore, we assumed that not only single RPSs but also the boundary regions that flank the RPSs are prerequisite for the expression of some biological functions. In this way, we recognized the necessity of examining the precise structure of the boundary regions of RPS. Since the hybridization signals derived from RPSs were the most intense for chromosome 6 , due to the presence of a large number of copies of the repeat in a limited region (Iwaguchi et al., 1992a) and also as only two Sfil fragments were detected (see below), we focused our attention on this chromosome. When chromosome $6(1400 \mathrm{kbp})$ was isolated by PFGE, digested by $S f \mathrm{I}$ and the resulting fragments separated by PFGE followed by Southern hybridization for detection of RPS signals, only bands of 760 and $510 \mathrm{kbp}$ and a band of the intact chromosome were obtained (Fig. 4). These three RPS-hybridizing bands also 
(a)

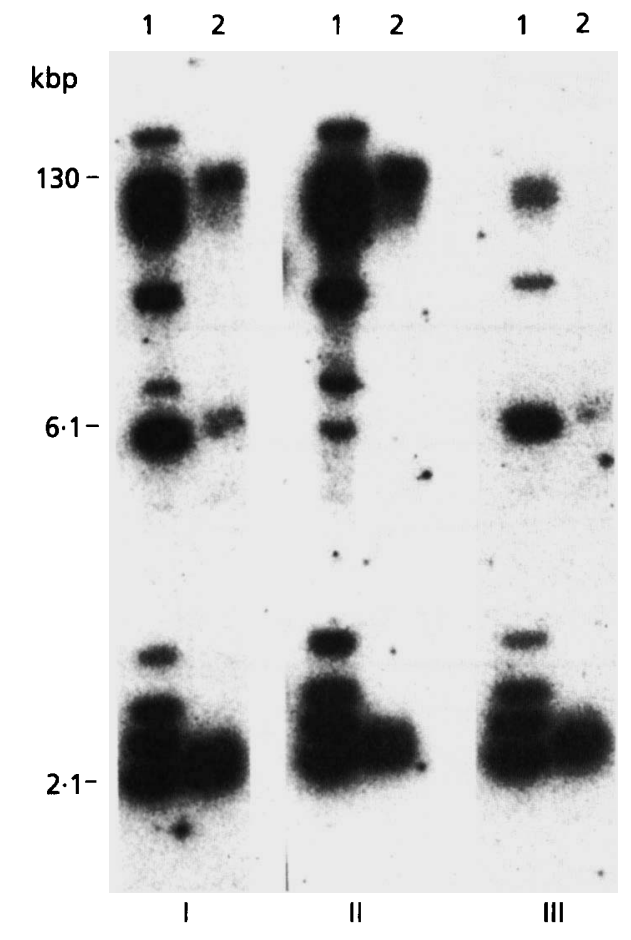

(b)

$$
\begin{array}{lllll}
1 & 2 & 3 & 4 & 5
\end{array}
$$

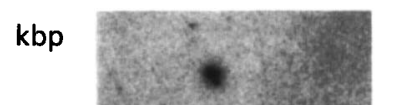

Fig. 5. Detection of the boundary regions of the RPS cluster on chromosome 6 . (a) Total chromosomal DNA (lane 1) and chromosome 6 (lane 2) of FC18 were prepared (see Methods), digested by Smal and fractionated by PFGE under ramping conditions from 20 to $100 \mathrm{~s}$ at $180 \mathrm{~V}$ for $15 \mathrm{~h}$ in $0.85 \%(w / v)$ agarose. Signals were detected using a ${ }^{32}$ p-labelled Pstl-Scal (probe A, I), Scal-Clal (probe B, II) or Clal-Pstl (probe C, III) fragment of pRPS101 (Chibana et al., 1994) as a probe. (b) Chromosome 6 of FC18 was digested with Smal alone (lane 1), or double-digested with Smal and Xhol (lane 2), Smal and BamHI (lane 3), Smal and Mlul (lane 4) or Smal and Nrul (lane 5). The digested samples were fractionated by PFGE under the same conditions as in (a). Signals were detected by Southern hybridization using ${ }^{32}$ p-labelled RPS1 (from pSI3-12) as a probe. The sizes indicated were calculated from the mobilities of marker DNAs ( $\lambda$ phage concatemers and $\lambda$ phage DNA digested by ECoT14 I). Data shown in the figure were from one set of experiments. Similar results were obtained in separate experiments. included telomeric sequences, as shown by hybridization with the pMM100 probe (Fig. 4, lane 3). This result indicated that no SfiI site was present in regions other than in the RPS-containing region, and that the RPS region was located in the middle of the chromosome or was flanked by the two fragments of 760 and $510 \mathrm{kbp}$. Furthermore, the pTK2-9-1 probe, which was used as a marker probe for chromosome 6 (Iwaguchi et al., 1990), hybridized with the $760 \mathrm{kbp}$ fragment, and the snc2 and snc 3 probes (B. Magee, personal communication) hybridized to the $510 \mathrm{kbp}$ fragment (data not shown). These results correspond to the results of experiments with chromosome 5 of the strain 1006 reported by Chu $e t$ al. (1993), where chromosome 5 of strain 1006 and chromosome 6 of FC18 hybridized to the same chromosome marker.

Since each of the restriction enzymes SmaI, PstI, and EcoRI have only one recognition site in the RPS sequence, we were able to estimate the size of the flanking sequences of the RPS when chromosome 6 was digested with one of these enzymes. Digestion of chromosome 6 from strain FC18 with $S m a I$ yielded three bands that hybridized with the RPS1 probe after PFGE. The fragments were estimated to be about 130, 6.1 and $2 \cdot 1 \mathrm{kbp}$ in size (Fig. 5b). Similar-sized fragments were also obtained from the homologues of chromosome 6 from other strains (data not shown). The signal of $2 \cdot 1 \mathrm{kbp}$ represented the RPS units themselves. The bands of 130 and $6.1 \mathrm{kbp}$ were assumed to be fragments derived from either one of the boundary regions that flanked the RPS: we postulated that the $130 \mathrm{kbp}$ band fragment contained one end of the
RPS and the $6.1 \mathrm{kbp}$ fragment contained the other end. To examine the validity of this assumption, a Pst $\mathrm{I}$ fragment from pRPS101 (Chibana et al., 1994) was divided into three small subunits, A (PstI-ScaI), B (ScaI-ClaI), and $\mathrm{C}(\mathrm{Cla}-\mathrm{I}-\mathrm{Pst} \mathrm{I})$, as shown schematically in Fig. 6(c), and then each was used as a probe for hybridization with the SmaI fragments of chromosome 6 (Fig. 5a). All three probes hybridized to the RPS units of $2.1 \mathrm{kbp}$. Probe A hybridized to both the $130 \mathrm{kbp}$ and $6.1 \mathrm{kbp}$ fragments. Probe B hybridized to the $130 \mathrm{kbp}$ but not to the $6.1 \mathrm{kbp}$ fragment, while probe $C$ did not hybridize to the $130 \mathrm{kbp}$ fragment but did to the $6.1 \mathrm{kbp}$ fragment. Assuming that RPS units are tandemly repeated in a limited region of each homologue of chromosome 6 (Iwaguchi et al., 1990), we deduced that the RPS region was flanked by $S \mathrm{maI}$ fragments of $130 \mathrm{kbp}$ and $6.1 \mathrm{kbp}$. To obtain more details about the physical maps of the RPS boundary regions, double digestion with $S m a \mathrm{I}$ and XhoI, BamHI, MluI or $\mathrm{NruI}$ was performed (Fig. 5b). Double digestion with $S m a I$ and Xhol gave a $66 \mathrm{kbp}$ band instead of a $130 \mathrm{kbp}$ band while the other signals at 6.1 and $2.1 \mathrm{kbp}$ were unchanged. This result indicates that an $X b o \mathrm{I}$ site was located within the $130 \mathrm{kbp} \mathrm{SmaI} \mathrm{fragment.} \mathrm{In} \mathrm{the} \mathrm{same}$ way, double digestions with $S m a \mathrm{I}$ and $B a m \mathrm{HI}, M l u \mathrm{I}$ or $\mathrm{NruI}$ produced RPS-positive bands of 27, 62 and $78 \mathrm{kbp}$, respectively, instead of the $130 \mathrm{kbp}$ band. These digestions did not affect the signals from the $6.1 \mathrm{kbp}$ and $2.1 \mathrm{kbp}$ bands, indicating that no recognition sites for these enzymes were present in the $6.1 \mathrm{kbp}$ and $2.1 \mathrm{kbp}$ fragments. From these results, a map of the entire structure of chromosome 6 was drawn, as shown in Fig. 6. 
(a)

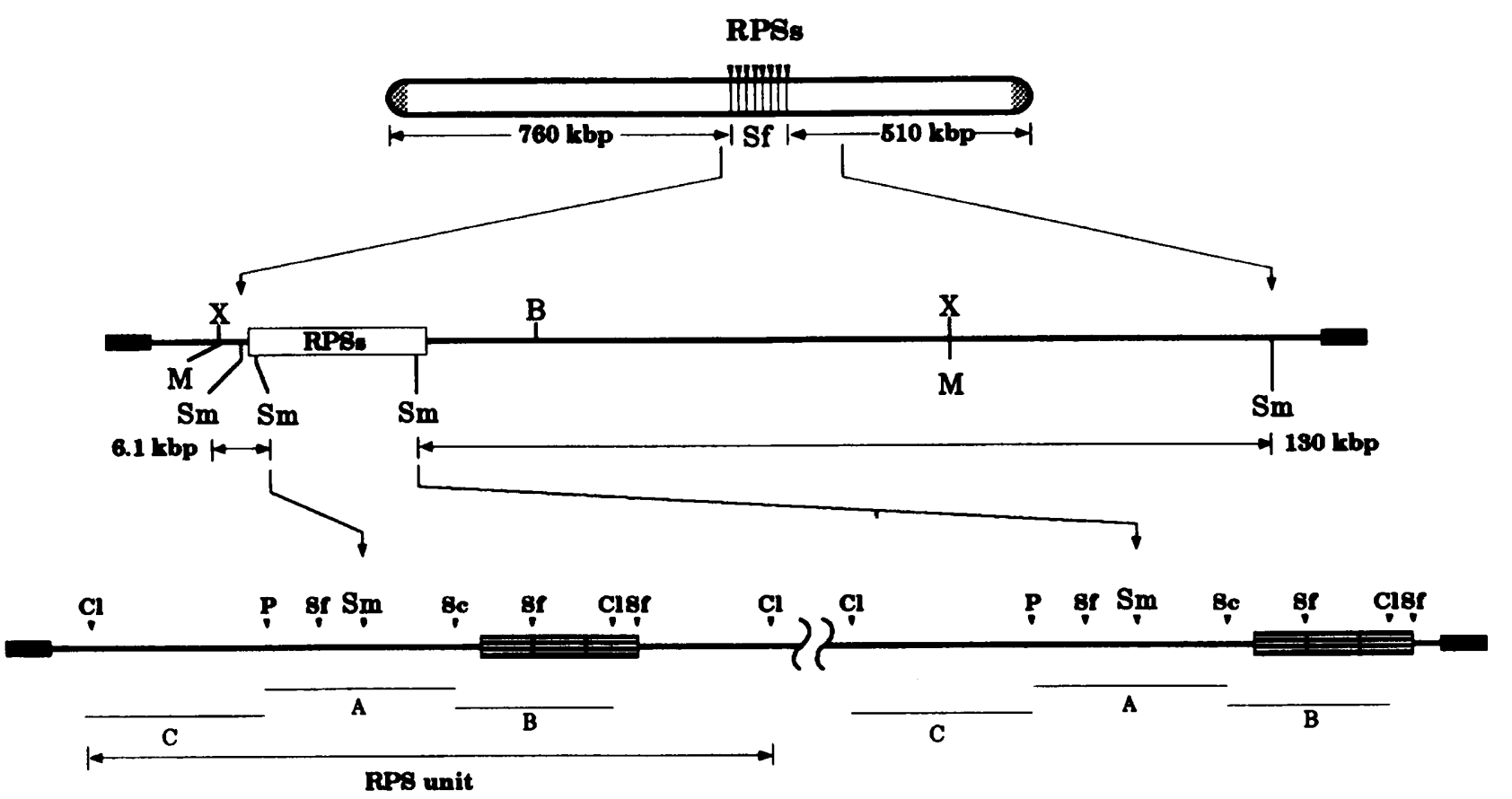

(b)

(c)

Fig. 6. Schematic representation of the physical map of chromosome 6 of C. albicans strain FC18 deduced following digestion with Sfil, Smal, Xhol, BamHI, Mlul and Nrul and probing with the RPS. The restriction sites are indicated as follows: B, BamHI; Cl, Clal; M, Mlul; N, Nrul; P, Pstl; Sc, Scal; Sf, Sfil; Sm, Smal; X, Xhol. (a) Overall structure of chromosome 6 from digestion with Sfil and probing with the RPS and telomeric sequences (see Fig. 3). The actual number of Sfil restriction sites within the RPSs was much greater than indicated here (see Discussion). The telomeric regions are indicated by shading (鮝). (b) Region around the RPS cluster, as determined by digestion with Smal, Xhol, BamHI, Mlul and Nrul and probing with RPS1 (see Fig. 5b). The double-headed arrows $(\leftrightarrow)$ indicate RPS boundaries flanked by Smal sites. The regions outside of the RPS boundary are shown by filled boxes $(\square)$. The Sfil recognition sites have been omitted. (c) Boundary regions of the RPS, as determined by hybridization with the sub-RPS probes (see Results, Fig. 5a). The regions of the sub-RPS probes are indicated by straight lines, and labelled A, B and C, respectively. The structure of RPS with short repetitive sequences [alt, hatched boxes (圆)] is included (Chibana et al., 1994). The lines with arrowheads indicate the outermost recognition sites of Smal in the RPS region. The double-headed arrows indicate one RPS unit. The repetitive RPS units have been omitted (- $\iint-$. Data shown in the figure were from one set of experiments. Similar results were obtained in separate experiments.

\section{DISCUSSION}

It has been estimated that the RPSs are present at a minimum of 60 copies in the diploid genome of $C$. albicans (Iwaguchi et al., 1992a). We showed here that RPSs are distributed in limited regions of all except one chromosome (Fig. 1). We could assign all the signals from the total chromosomal DNA (Fig. 1, lane Total) to those from each chromosome separately (Fig. 1, lanes $1+2$ to 8). Although the numbers of copies seemed to differ among the chromosomes, as indicated by the different intensities of the various signals, RPSs were located in clusters and were not dispersed on chromosomes with multiple copies of RPS (Fig. 2). Furthermore, by digestion of each chromosome with $S f \mathrm{I}$, we were able to detect small-sized fragments derived from RPSs (Fig. 3). These fragments corresponded to those of the already cloned RPSs (Chibana et al., 1994). This result demonstrates the presence of conserved Sfil fragments in the RPS region, suggesting that $S f i$ recognition sites in the RPS region are conserved. As discussed below, if the RPS is related to some biological function, acting for example as a centromere, all the chromosomes would have to carry the
RPS regardless of the location on the chromosome. However, we failed to obtain any RPS-specific signals from chromosome 4 under various experimental conditions, whereas the other chromosomes always gave significant signals. Nonetheless, it remains unknown whether chromosome 4 lacks a portion of the RPS.

Recently, Chu et al. (1993) constructed a macro-restriction map of the chromosomes of $C$. albicans by using a rarecutting enzyme, Sfil, and Southern hybridization with specific gene probes for two strains, 1006 and WO-1. They found that some of the $S$ fiI chromosomal fragments had been translocated to different chromosomes and they suggested that Sfil sites might be the break points responsible for translocation of the fragments. It is noteworthy that the RPS units contained several sites for $S f i I$. In fact, we detected small SfiI fragments derived from the RPS of each chromosome except for chromosome 4 (Fig. 3). Hence, chromosome 6, which contains at least 10 tandemly repeated RPS units (Iwaguchi et al., 1992a), should have more than 30 Sfi sites, which could be junction sites for translocation. The finding that these sites were clustered in a limited region(s) on almost all 
chromosomes (see Fig. 1) tends to substantiate the speculation by Chu et al. (1993).

Among the repeated sequences in C. albicans reported to date, some features associated with Rel-2 are very similar to those of RPS (Thrash-Bingham \& Gorman, 1993). The Rel-2 sequence is about $2.7 \mathrm{kbp}$ in length, it hybridizes to every chromosome, and it includes subrepeat sequences of $29 \mathrm{bp}$. RPS varies in size from 1.9 to $2.7 \mathrm{kbp}$ in increments of $0.2 \mathrm{kbp}$, the RPS probe hybridizes to almost every chromosome, and it includes an inner repeated sequence, COM29, of 29 bp. Additionally, both probes hybridize not only to genomes of all strains of $C$. albicans tested but also to that of Candida stellatoidea (ThrashBingham \& Gorman, 1993; A. Chindamporn, data not shown) although the intensity of the signals in the case of C. stellatoidea was weaker than that for C. albicans. However, no sequence homology was detected between the two sequences (Chibana et al., 1994). The structural similarity suggests, however, that some function might be partly shared by both sequences despite differences in nucleotide sequence.

Since the RPSs are located in limited regions of all chromosomes apart from chromosome 4 (Fig. 1), they might have some important role in chromosome function. Recently, we found that the RPS includes many repeats of the short sequence, alt, and we suggested a possible centromeric function by analogy to the structural features of human alpha-satellite DNA (Chibana et al., 1994). Most of the physically characterized centromeres include inner repetitive sequences in their higher-order units (Willard \& Waye, 1987; Clarke, 1990; Takahashi et al., 1992; Vogt, 1992; Centola \& Carbon, 1994). If the RPS functions as a centromere, a plasmid that includes autonomously replicating sequence (ARS) for C. albicans, a selectable marker such as $\mathrm{Ura}^{+}$, and the RPS, would be expected to be maintained in $\mathrm{Ura}^{-}$cells as a stable episome. However, as mentioned in Results, no cells transformed with an RPS-carrying plasmid were stable during mitosis. Therefore, we postulated that not only the RPS sequence but also the boundaries of the RPS might be required for expression of some important role in cellular function. To examine this possibility, we physically mapped an extended region that included the RPSs on chromosome 6 . Chromosome 6 could be used as a model chromosome that carries only one set of boundary regions of the RPSs since only two Sfil fragments were detected. After digestion with $S m a I$ three fragments that hybridized with an RPS probe were obtained: that of $2 \cdot 1 \mathrm{kbp}$ represented the RPS units and the other two, of $130 \mathrm{kbp}$ and $6 \cdot 1 \mathrm{kbp}$, were inferred to be the junction fragments and to contain RPS fragments at their respective ends. The $130 \mathrm{kbp}$ fragment was derived from the $510 \mathrm{kbp}$ fragment generated by digestion of chromosome 6 by Sfil, and the $6.1 \mathrm{kbp}$ fragment was derived from the $760 \mathrm{kbp}$ Sfil fragment, as shown by SmaI digestion followed by hybridization with an RPS probe of the respective $S$ fi fragments (data not shown). To determine which part of the RPS was adjacent to the $6.1 \mathrm{kbp}$ and the $130 \mathrm{kbp}$ fragments, hybridizations using sub-RPS probes were performed (Fig. 5a). From our results, we could demonstrate a characteristic structure of the boundary region of RPS units which were tandemly arrayed on this chromosome.

\section{ACKNOWLEDGEMENTS}

The authors thank Dr B. B. Magee of the University of Minnesota for kindly providing the snc2 and snc 3 probes, and Dr M. J. McEachern of the University of California, San Francisco, for the $\mathrm{Ca} 7$ probe DNA. The authors also thank Ms I. Mizuguchi for expert photographic assistance. This work was supported by grants-in-aid for scientific research from the Ministry of Education, Science and Culture, Japan. A.C. is the recipient of a scholarship from the Hitachi Foundation.

\section{REFERENCES}

Anderson, J., Srikantha, T., Morrow, B., Miyasaki, S. H., White, T. C., Agabian, N., Schmid, J. \& Soll, D. R. (1993). Characterization and partial nucleotide sequence of the DNA fingerprinting probe Ca3 of Candida albicans. J Clin Microbiol 31, 1472-1480.

Barton, R. C. \& Scherer, S. (1994). Induced chromosome rearrangements and morphologic variation in Candida albicans. $J$ Bacteriol 176, 756-763.

Centola, M. \& Carbon, J. (1994). Cloning and characterization of centromeric DNA from Neurospora crassa. Mol Cell Biol 14, 1510-1519.

Chibana, H., Iwaguchi, S. -I., Homma, M., Chindamporn, A., Nakagawa, Y. \& Tanaka, K. (1994). Diversity of tandemly repetitive sequences due to periodic repetitions in the chromosomes of Candida albicans. J Bacteriol 176, 3851-3858.

Chindamporn, A., Iwaguchi, S. -I., Nakagawa, Y., Homma, M. \& Tanaka, K. (1993). Clonal size-variation of rDNA cluster region on chromosome XII of Saccharomyces cerevisiae. J Gen Microbiol 139, 1409-1415.

Chu, W.-S., Magee, B. B. \& Magee, P. T. (1993). Construction of an $S$ fil macrorestriction map of the Candida albicans genome. J Bacteriol 175, 6637-6651.

Clarke, L. (1990). Centromeres of budding and fission yeasts. Trends Genet 6, 150-154.

Doi, M., Homma, M., Chindamporn, A. \& Tanaka, K. (1992). Estimation of chromosome number and size by pulsed-field gel electrophoresis (PFGE) in medically important Candida species. $J$ Gen Microbiol 138, 2243-2251.

Iwaguchi, S. -I., Homma, M. \& Tanaka, K. (1990). Variation in the electrophoretic karyotype analysed by the assignment of DNA probes in Candida albicans. J Gen Microbiol 136, 2433-2442.

Iwaguchi, S. -I., Homma, M., Chibana, H. \& Tanaka, K. (1992a). Isolation and characterization of a repeated sequence (RPS1) of Candida albicans. J Gen Microbiol 138, 1893-1900.

Iwaguchi, S. -I., Homma, M. \& Tanaka, K. (1992b). Clonal variation of chromosome size derived from the rDNA cluster region in Candida albicans. J Gen Microbiol 138, 1177-1184.

Kurtz, M. B., Cortelyou, M. W., Miller, S. M., Lai, M. \& Kirsch, D. R. (1987). Development of autonomously replicating plasmids for Candida albicans. Mol Cell Biol 7, 209-217.

Lasker, B. A., Carle, G. F., Kobayashi, G. S. \& Medoff, G. (1989). Comparison of the separation of Candida albicans chromosome-sized 
DNA by pulsed-field gel electrophoresis techniques. Nucleic Acids Res 17, 3783-3793.

Lasker, B. A., Page, L. S., Lott, T. J., Kobayashi, G. S. \& Medoff, G. (1991). Characterization of CARE-1: Candida albicans repetitive element-1. Gene 102, 45-50.

Lott, T. J., Boiron, P. \& Reiss, E. (1987). An electrophoretic karyotype for Candida albicans reveals large chromosomes in multiples. Mol \& Gen Genet 209, 170-174.

Magee, B. B. \& Magee, P. T. (1987). Electrophoretic karyotypes and chromosome numbers in Candida species. J Gen Microbiol 133, 425-430.

Magee, B. B., Koltin, Y., Gorman, J. A. \& Magee, P. T. (1988). Assignment of cloned genes to the seven electrophoretically separated Candida albicans chromosomes. Mol Cell Biol 8, 4721-4726.

McEachern, M. J. \& Hicks, J. B. (1993). Unusually large telomeric repeat in the yeast Candida albicans. Mol Cell Biol 13, 551-560.

Rustchenko-Bulgac, E. P., Sherman, F. \& Hicks, J. B. (1990). Chromosomal rearrangements associated with morphological mutants provide a means for genetic variation of Candida albicans. J Bacteriol 172, 1276-1283.

Sadhu, C., McEachern, M. J., Rustchenko-Bulgac, E. P., Schmid, J., Soll, D. R. \& Hicks, J. B. (1991). Telomeric and dispersed repeat sequences in Candida yeasts and their use in strain identification. $J$ Bacteriol 173, 842-850.

Scherer, S. \& Stevens, D. A. (1988). A Candida albicans dispersed, repeated gene family and its epidemiologic applications. Proc Natl Acad Sci US A 85, 1452-1456.

Schwartz, D. C. \& Cantor, C. R. (1984). Separation of yeast chromosome-sized DNAs by pulsed field gradient gel electrophoresis. Cell 37, 67-75.

Suzuki, T., Kobayashi, I., Kanbe, T. \& Tanaka, K. (1989). Highfrequency variation of colony morphology and chromosome reorganization in the pathogenic yeast Candida albicans. $J$ Gen Microbiol 135, 425-434.

Takahashi, K., Murakami, S., Chikashige, Y., Funabiki, H., Niwa, O. \& Yanagida, M. (1992). A low copy number central sequence with strict symmetry and unusual chromatin structure in fission yeast centromere. Mol Biol Cell 3, 819-835.

Thrash-Bingham, C. \& Gorman, J.A. (1993). Identification, characterization and sequence of Candida albicans repetitive DNAs Rel-1 and Rel-2. Curr Genet 23, 455-462.

Vogt, P. (1992). Code domains in tandem repetitive DNA sequence structures. Chromosoma 101, 585-589.

Willard, H. F. \& Waye, J. S. (1987). Hierarchical order in chromosome-specific human alpha satellite DNA. Trends Genet 3, 192-198.

Received 1 August 1994; revised 22 September 1994; accepted 19 October 1994. 